\title{
Mountain-bike racing - the influence of prior glycogen- reducing exercise and glutamine supplementation on selected stress and immune parameters
}

\author{
C Smith (PhD (Physiological Sciences)) \\ K H Myburgh (PhD (Physiology)) \\ Department of Physiological Sciences, Stellenbosch University, W Cape
}

\begin{abstract}
Objective. To investigate the effect of pre-exercise glutamine supplementation and the influence of a prior acute bout of glycogen-reducing exercise on the general stress and immune response to acute high-intensity cycling.
\end{abstract}

Design. Randomised, double-blind, cross-over supplementation study.

Setting and intervention. Subjects performed a series of 4 simulated mountain-bike races lasting $\approx 60$ minutes each on separate days 1 week apart, with/ without prior glycogen-reducing exercise on a known outdoor course with/ without pre-exercise glutamine supplementation. Blood samples were collected pre- and immediately post-exercise after each race.

Main outcome measures. Circulating concentrations of cortisol (COR) and dehydroepiandrosterone-sulphate (DHEAS) were assessed at all time points, as well as changes in white blood cell (WBC) subpopulation distribution.

Results. COR was elevated in all groups post-exercise $(p<0.0001)$, but neither glycogen reduction, nor glutamine supplementation had any effect. DHEAS increased post-exercise $(p<0.05)$, with a greater relative increase in glutamine-supplemented subjects $(p=0.07)$. Total WBC and neutrophil counts in all groups were elevated after exercise (both $p<0.0005$ ). Glutamine supplementation had no effect on differential WBC counts or distribution, but total WBC $(p=0.06)$ and monocyte $(p<0.05)$ counts showed greater increases after glycogen reduction. Gluta-

\section{CORRESPONDENCE:}

C Smith

Department of Physiological Sciences

Stellenbosch University

Private Bag X1

Matieland

7602

Tel: 021-808 4388

Fax: 021-808 3145

E-mail: csmith@sun.ac.za mine supplementation was associated with greater postexercise decreases in $C D 4^{+}$count $(p=0.07)$ and $C D 4^{+}$: $\mathrm{CD}^{+}$ratio $(p=0.01)$ after glycogen-reducing exercise.

Conclusions. We conclude that pre-exercise glutamine supplementation may have an anticortisol effect by enhancing the DHEAS response to exercise stress. The suppressive effect of glutamine supplementation on $\mathrm{CD}^{+}$: $\mathrm{CD}^{+}$ratio and its positive effect on monocyte count after repeated bouts of exercise warrants further investigation.

\section{Introduction}

Glutamine is primarily synthesised and stored in skeletal muscle and the lungs and is the principal metabolic fuel for the small intestine enterocytes, and for lymphocytes, macrophages, and fibroblasts. ${ }^{16}$ Under conditions of severe stress (such as trauma, burns, surgery or sepsis), ${ }^{2,13}$ these rapidly replicating cells exhibit accelerated use of glutamine, ${ }^{2}$ which may result in a $35-50 \%$ decrease in plasma glutamine concentrations that lasts for several days. ${ }^{22}$

Similarly, acute, prolonged, exhaustive exercise resulted in a $\approx 25 \%$ decrease in plasma glutamine concentration, which lasted for 6 - 9 hours after a marathon. ${ }^{4}$ Reports of decreased plasma glutamine levels in overtrained athletes at rest ${ }^{23,27}$ suggest that long-term chronic exercise stress may result in a chronically depressed plasma glutamine concentration. In the absence of exercise, cortisol infusions in humans resulted in decreased circulating glutamine concentration after 8 hours, ${ }^{13}$ indicating that increased glucocorticoid levels are an important factor influencing glutamine turnover. Considered together, these 4 studies indicate the following: firstly, that exercise stress increases glutamine utilisation rate, secondly that glutamine stores are not readily available during or after exercise, and thirdly that these effects may be mediated by cortisol. Much evidence exists for the negative effects of long-duration endurance exercise on both the non-specific and the specific immune system, including decreased lymphocyte proliferative ability, impaired neutrophil function, impaired antibody synthesis and a decreased $\mathrm{CD}^{+}: \mathrm{CD}^{+}$ ratio. ${ }^{17,19,21,29,30}$ Together these results suggest a possible link between decreased availability of glutamine after exercise stress exposure and post-exercise immune suppression.

However, this 'glutamine hypothesis' has recently been contested. ${ }^{14,37}$ The majority of in vitro studies illustrating 
suppressed immune capacity used concentrations of glutamine below $100 \mu \mathrm{M}^{24,26}$ However, serum glutamine concentration decreases by only $\approx 10-20 \%$ after strenuous exercise, so that post-exercise glutamine levels are usually maintained at a level in excess of $400 \mu \mathrm{M}$. ${ }^{14}$ Furthermore, while oral glutamine supplementation during the last 30 minutes of a 2-hour exercise bout (laboratory-based cycling) attenuated the decrease in plasma glutamine concentration, it had no effect on the exercise-induced decrease in lipopolysaccharide (LPS)-induced neutrophil elastase release or PMA-stimulated neutrophil oxidative burst capacity. ${ }^{36}$ These studies suggest that plasma glutamine concentration is unlikely to play an important role in the maintenance of the immune response to exercise stress.

Despite the recent contention that glutamine is not the link' responsible for exercise-induced immune suppression, ${ }^{14}$ a gap exists in the literature with regard to the timing of glutamine supplementation. Most in vivo studies reporting no effect of glutamine supplementation administered glutamine either post-exercise ${ }^{25}$ or during the later stages of exercise, ${ }^{36}$ in other words at a time when immune cell function is already compromised. Recently, administration of a single dose of glutamine prior to an acute high-intensity exercise bout, was reported to prevent decreases in post-exercise glutamine levels in female runners. ${ }^{34}$ It is therefore possible that the timing of glutamine supplementation may be an important factor influencing its effect in vivo.

Given the reported involvement of cortisol in glutamine metabolism, ${ }^{13}$ it is possible that pre-exercise glutamine supplementation may alter the cortisol response to acute exercise. In turn, given the known negative effects of cortisol on inflammation and $\mathrm{T}_{\mathrm{H}}$ 1-mediated immune function, ${ }^{6,8,15}$ an effect of glutamine on immune function may not be direct, but only discernible in the context of its influence on endocrine parameters. Since the cortisol antagonist dehydroepiandrosterone (DHEA) is known to have an opposing effect to cortisol on immune function, 7,33 assessment of this parameter in conjunction with cortisol should provide a more complete picture of possible beneficial effects of glutamine supplementation.

Therefore, the main purpose of this study was to determine the effect of pre-exercise oral glutamine supplementation on: (i) the general endocrine stress response to an acute bout of high-intensity cycling exercise simulating competition; and (ii) changes in immune cell distribution as a result of this exercise. A second purpose was to investigate the response of these parameters for the same simulated competition, but under more extreme conditions, by inducing low glycogen levels with an acute exercise bout 1 day prior to the simulated competition.

\section{Methods}

\section{Subject recruitment.}

Eight healthy, sub-elite, competitive male off-road cyclists were recruited. Prior to this study, all participants had taken part in at least 1 competitive cross-country mountain bike league event during the racing season in which the study took place. Smokers, as well as individuals taking daily vitamins or other dietary supplements, were excluded from the study. Subjects were instructed to refrain from using any supplements for the duration of the protocol. Informed, written consent was obtained from all volunteers before admittance into the study. The study protocol was approved by the Stellenbosch University Ethics Committee.

\section{Exercise intervention}

This double-blind, cross-over design study consisted of 4 simulated mountain bike races, each separated by 1 week. The race took place on a known mountain biking route with a total distance of $20 \mathrm{~km}$. Due to logistic difficulty, the exact racing times for each participant could not be recorded. However, all races were $\approx 60$ minutes in duration, which is in accordance with race times recorded for actual competitive events on the same route. For each race, subjects were required to arrive at the laboratory fasted at $08 \mathrm{~h} 00$. A blood sample was collected, after which each subject received $300 \mathrm{ml}$ of either a glutamine drink (low-calorie drink plus $5 \mathrm{~g}$ glutamine) or placebo (low-calorie drink only). Subjects warmed up doing an easy $10 \mathrm{~km}$ road cycle to the race venue, and competed in a simulated race that started 30 minutes after taking the glutamine/placebo drink. (Plasma glutamine concentration was previously reported to double within 30 minutes after a supplementation protocol similar to the one used in our study. ${ }^{3}$ ) Immediately after finishing the race, another blood sample was obtained. For 2 of the 4 races, normal repletion of glycogen stores was achieved by subjects refraining from training or any other form of exercise for at least 24 hours before the race, as well as eating a carbohydrate-rich supper the evening before the race. Two hours of cycling exercise at $\approx 64 \% \mathrm{VO} 2_{\text {max }}$ was previously shown to result in an average decrease in muscle glycogen content from 80 to $18 \mathrm{mmol}$ glucose units/ kg muscle wet weight. ${ }^{11}$ Taking this protocol into account, for the other 2 races, a significant reduction in glycogen levels was induced by a 3-hour late afternoon cycle while drinking water only, followed by a low-carbohydrate supper the evening before the race. Although portion size and quantity were not specified, a list of foods from which to choose was supplied for both meal types, and subjects were instructed not to deviate from this. All races were randomised for glutamine/placebo, but not for glycogen depletion/control. This was done to ensure equal relative competitiveness and motivation of all subjects during all races, especially for races 1 day after glycogen-depleting exercise.

\section{Sample collection}

Blood was obtained by venepuncture of a forearm vein by experienced phlebotomists at all time points. Whole blood was collected in BD vacutainers (Preanalytical Solutions, Plymouth, UK). EDTA-anticoagulated blood samples were collected for total and differential white blood cell (WBC) count and haematocrit determination. Lithium heparin-anticoagulated blood samples were collected for determination of T-cell subpopulations. A third blood sample, collected in a SST vacutainer, was allowed to clot at room temperature for 


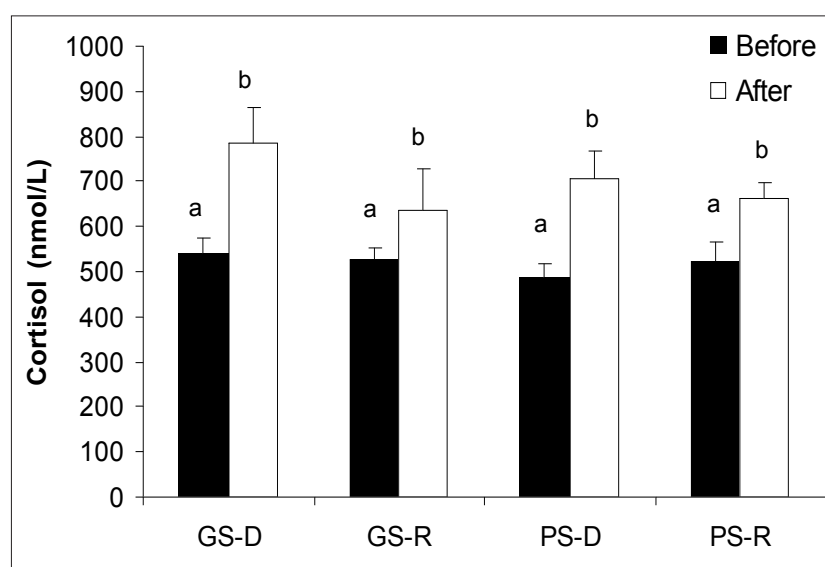

Statistical analysis: main effects ANOVA indicated significant effect of exercise $(p<0.0001)$, but no effect of either glutamine supplementation or glycogen reduction. Bonferroni's post hoc analysis showed no significant difference between groups at any time point. Error bars indicate SEM. (GS-D = glutamine-supplemented, prior glycogen-reducing exercise; GS-R = glutamine-supplemented, glycogen-replete and rested; PS-D = placebo-supplemented, prior glycogenreducing exercise; PS-R = placebo-supplemented, glycogen-replete and rested.)

Fig. 1. The effect of prior glycogen-reducing exercise and glutamine supplementation on the average cortisol response to $\approx 60$ minutes of simulated mountain bike racing.

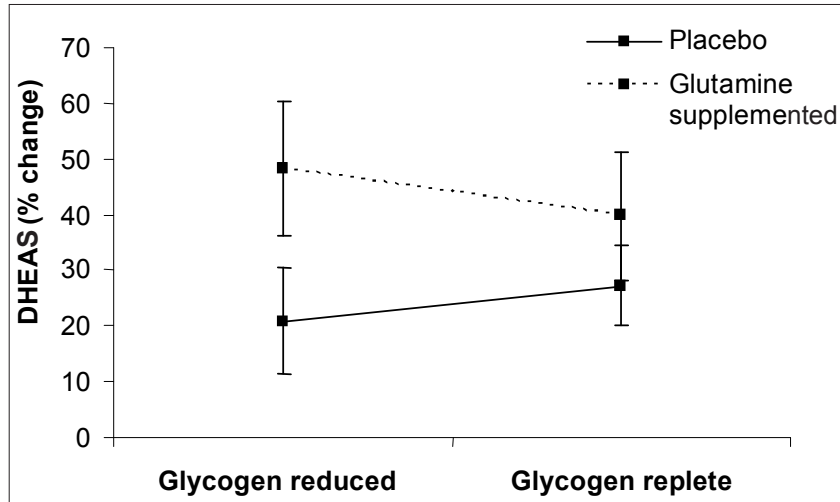

Statistical analysis: ANOVA tendency for a main effect of glutamine supplementation on the DHEAS response to exercise $(p=0.07)$, but no effect of prior glycogen-reducing exercise. Bonferroni's post hoc analysis: No significant difference between groups. Error bars indicate SEM.

Fig. 2. The effect of glutamine supplementation and prior glycogen-reducing exercise on the relative DHEAS response to $\approx 60$ minutes of simulated mountain bike racing.

10 minutes, centrifuged at $3000 \mathrm{rpm}$ for 10 minutes at $4^{\circ} \mathrm{C}$, and frozen at $-80^{\circ} \mathrm{C}$ for subsequent batch analysis of serum cortisol and DHEA-sulphate (DHEAS) concentrations.

\section{Sample analysis}

EDTA-anticoagulated blood samples were kept at room temperature and analysed within 4 hours after collection for total and differential WBC counts, as well as haematocrit, by automated analysis (Coulter STKS, Beckman/Coulter, Fullerton, Calif.). Heparinised blood samples were also kept at room temperature and analysed within 4 hours for T-cell subpopulations using standard 3-colour flow cytometry. Automated analysers were used for determination of serum cortisol (Access B module 81600, Beckman/Coulter, Fullerton, Calif.) and DHEAS (Immulite I, Diagnostic Products Corporation, Los Angeles, Calif.) concentrations.

\section{Statistical analysis}

All results are presented as means \pm standard error of the mean (SEM). Effects of glutamine supplementation and prior exercise on parameters investigated, were assessed using repeated measures factorial analysis of variance (ANOVA). Differences between groups were determined using Bonferroni's post hoc tests. Level of significance was set at $p<$ 0.05 .

\section{Results}

Two athletes competed in only 1 race each, after which they withdrew from the study due to injuries unrelated to the study protocol. The data obtained for these 2 subjects were excluded from the analyses illustrated below. All subjects were questioned about episodes of illness in the week prior to each race to prevent illness from confounding results obtained. Only 1 subject reported such an episode (sore throat for 2 days which was treated only for symptoms). In this case, the subject was not allowed to take part in the study protocol for a period of 1 week. Due to problems not related to the study, 3 other subjects also failed to take part in 1 of the simulated races. Therefore, these 4 subjects together took part in a fifth race, 1 week after the last race, to finish the protocol.

Average age, height and body mass of the participants were $28 \pm 5$ years, $180 \pm 3 \mathrm{~cm}$ and $76 \pm 7 \mathrm{~kg}$ respectively. Haematocrit values changed significantly from before to after exercise $(0.45 \pm 0.003$ v. $0.46 \pm 0.004, p<0.01)$. Therefore, all post-exercise values were adjusted according to the percentage change in haematocrit, to correct for changes in plasma volume, prior to statistical analysis.

A main effect of racing exercise was an increase in cortisol concentration from baseline $(p<0.0001$, Fig. 1$)$, which was independent of both glutamine supplementation and prior glycogen-reducing exercise. DHEAS concentration was also increased as a result of racing exercise (before $10.7 \pm 0.9 \mathrm{v}$. after $14.0 \pm 1.1 \mathrm{umol} / \mathrm{l}, p<0.05$ ). Glutamine supplementation was associated with a tendency for greater relative change in DHEAs concentration in response to racing exercise, independent of prior glycogen-reducing exercise $(p=0.07$, Fig. 2). However, the cortisol: DHEAS ratio was not different between treatment groups (main effect of treatment: $p=$ 0.97). 
All WBC counts are summarised in Table I. A main effect of racing exercise was a significantly increased overall total WBC count (before $5.38 \pm 0.27 \mathrm{v}$. after $8.87 \pm 0.89 \times 10^{3}$ cells/ul, $p<0.0005$ ) which was mainly due to a significantly increased neutrophil count (before $2.88 \pm 0.23 \mathrm{v}$. after $6.12 \pm$ $0.81 \times 10^{3}$ cells/ul, $\left.p<0.0005\right)$, while the changes in monocyte (before $0.51 \pm 0.02 \mathrm{v}$. after $0.59 \pm 0.05 \times 10^{3}$ cells $/ \mathrm{ul}, p=0.14$ ) and total lymphocyte (before $1.80 \pm 0.12 \mathrm{v}$. after $2.03 \pm 0.18 \mathrm{x}$ $10^{3}$ cells/ul, $p=0.42$ ) counts were not significant.

While the racing exercise-induced changes in neutrophil and total lymphocyte counts were independent of both glutamine supplementation and previous glycogen-reducing exercise, there was a main effect of glycogen-reducing exercise on the response of WBC $(p=0.06)$ and monocytes $(p=0.05)$ to racing exercise, with greater increases in cell count after glycogen-reduction than in the rested, glycogenreplete state, independent of glutamine supplementation (Fig. 3).

Although the total lymphocyte count did not change significantly as a result of simulated racing exercise, with no apparent effect of either glutamine supplementation or prior glycogen-reducing exercise, a clearer picture is obtained by considering changes in T-lymphocyte subpopulation distribution. Racing exercise had a significant main effect on relative $\mathrm{CD}^{+}$cell count, which decreased significantly after exercise (before $40.7 \pm 1.1$ v. after $36.5 \pm 1.0 \%, p<0.005$ ) Furthermore, while the percentage of $\mathrm{CD}^{+}$cells relative to the total lymphocyte count was not significantly influenced by racing exercise, glutamine supplementation or prior glycogenreducing exercise ( $p=0.11$, Fig. $4 a)$, there was a tendency for an interaction effect of glutamine supplementation and glycogen reduction on the relative $\mathrm{CD} 4^{+}$cell $(p=0.07$, Fig. $4 b)$ response to racing exercise. This resulted in a significant interaction effect of glutamine supplementation and glycogen reduction on the $C D 4^{+}: C D 8^{+}$ratio $(p=0.01$, Fig. $4 c)$, so that the decrease in $\mathrm{CD}^{+}: \mathrm{CD} 8^{+}$ratio was significantly greater when glutamine-supplemented when compared to placebo, after glycogen reduction ( $p<0.05$, Fig. 4c), but with no apparent differences when rested and glycogen-replete. However, despite this decrease, average $\mathrm{CD} 4^{+}: \mathrm{CD} 8^{+}$ratio remained within the expected range for athletes throughout the protocol (before $1.84 \pm 0.12 \mathrm{v}$. after $1.62 \pm 0.09$ )

\section{Discussion}

Our results illustrate the following main findings: (i) that in the presence of prior glycogen-reducing exercise glutamine supplementation results in greater increases from baseline in total WBC and monocyte counts after simulated racing exercise; (ii) that glutamine supplementation may play a role in increasing DHEAS levels in a stress situation irrespective of glycogen availability; and (iii) that glutamine supplementation affects the relative distribution of T-lymphocyte subpopulations in response to exercise under competition conditions with insufficient glycogen availability.

Mountain biking was recently characterised as an intermittent high-intensity endurance sport, with heart rate during a race ranging from 165 to 205 beats per minute

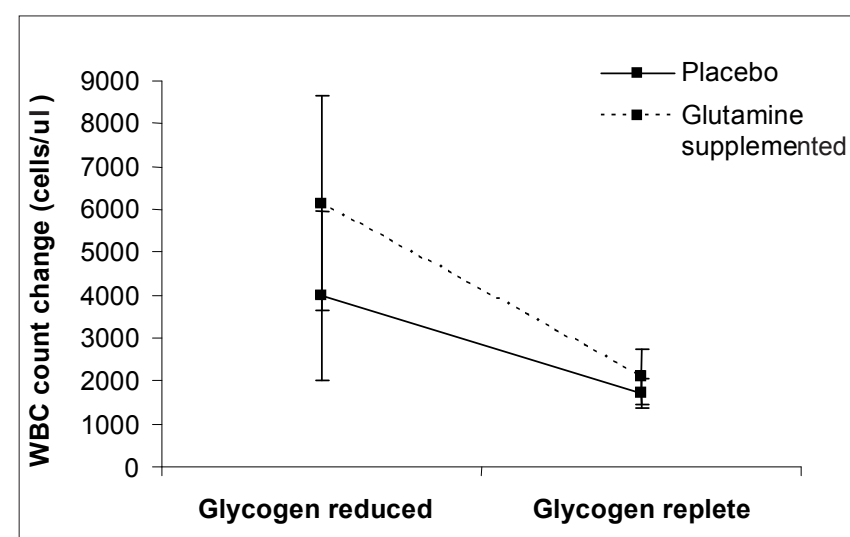

a) Statistical analysis: Main effects ANOVA indicates a tendency for a main effect of prior glycogen-reducing exercise $(p=0.06)$. Bonferroni's post hoc analysis: No significant difference between groups. Error bars are SEM.

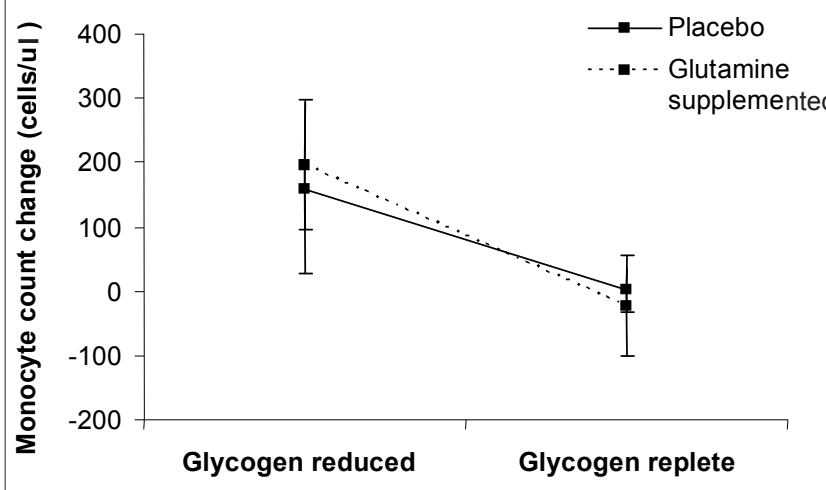

b) Statistical analysis: Main effects ANOVA indicates a significant effect of prior glycogen-reducing exercise $(p$ $=0.05)$. Bonferroni's post hoc analysis: No significant difference between groups. Error bars are SEM.

Fig. 3. The effects of glutamine supplementation and prior glycogen-reducing exercise on a) total WBC and b) monocyte responses, to $\approx 60$ minutes of simulated mountain bike racing.

(bpm) (mean of $91 \%$ of maximum heart rate previously assessed in a laboratory setting). ${ }^{32}$ Due to the nature of the terrain there are great fluctuations in power output (range 50 - $400 \mathrm{~W}$ ) for male participants in national and international racing events. ${ }^{32}$ While running and road cycling require a more or less constant, continuous effort, mountain biking therefore displays a succession of very high and lower power requirements, necessitating bursts of both power and endurance, and therefore both anaerobic and aerobic fitness. In agreement with earlier studies of endurance athletes, $9,10,18$ cortisol increased from baseline to post-exercise.

However, in addition our results also suggest an indirect anticortisol effect of glutamine, since glutamine-supplemented groups exhibited greater increases in DHEAS concentrations in response to exercise, compared with placebo groups 


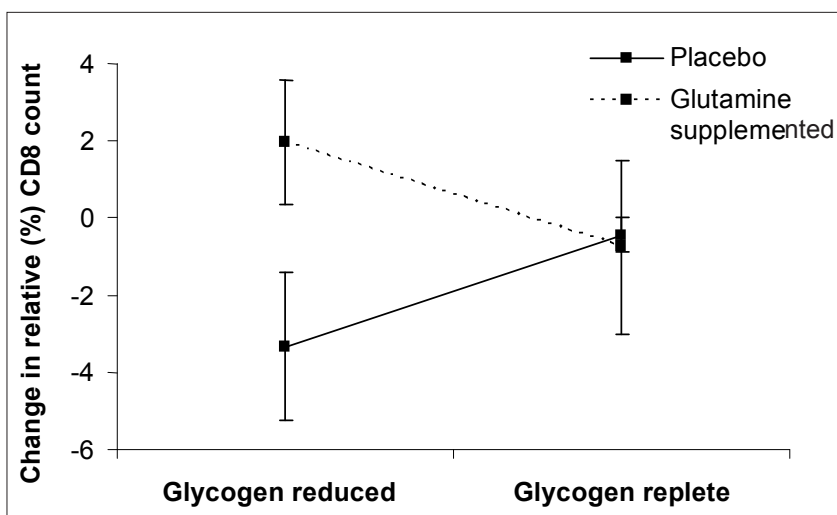

a) Statistical analysis: Main effects ANOVA showed no effect of exercise, glutamine supplementation, prior glycogenreducing exercise or an interaction between two or more predictors. Bonferroni's post hoc analysis showed no difference between groups. Error bars indicate SEM.

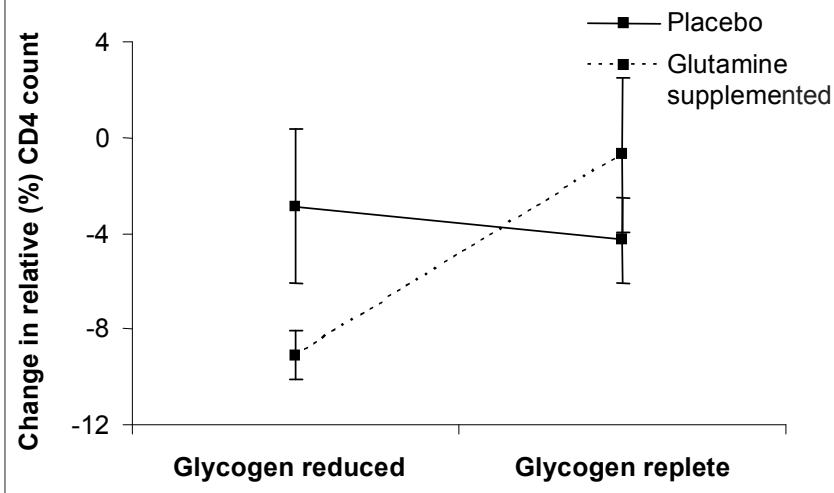

b) Statistical analysis: Main effects ANOVA showed a main effect of exercise $(p<0.005)$ and a tendency for an interaction effect of glutamine supplementation and prior glycogen-reducing exercise ( $p=0.07$ ), but no effect of glutamine supplementation or prior glycogen-reducing exercise alone. Bonferroni's post hoc analysis showed no difference between groups. Error bars indicate SEM.

(Fig. 2). Glutamine is known to play a role in amino acid synthesis, $^{16}$ and both glutamine and testosterone were reported to prevent glucocorticoid-induced muscle atrophy in animal and human models. ${ }^{28}$ Our result suggests that glutamine-enhanced DHEAS synthesis may be a possible mechanism for this beneficial effect, via an increased capacity for testosterone production. Maintenance of DHEAS levels is also associated with positive outcomes in situations of increased stress, ${ }^{1,7}$ while chronic decreased serum DHEAS concentration is associated with progression of various chronic diseases. ${ }^{5,12,35,38}$ It is therefore of importance to investigate this potentially beneficial effect of glutamine more comprehensively in this and other appropriate models of stress.

Our result of an effect of repeated exercise bouts on total WBC and monocyte count is in partial agreement with the association reported between the magnitude of increase in

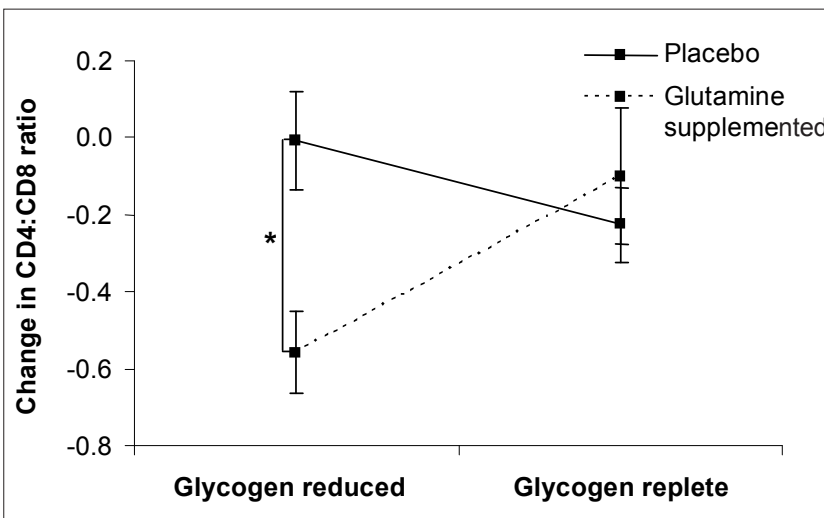

c) Statistical analysis: Main effects ANOVA showed a significant interaction effect of glutamine supplementation and prior glycogen-reducing exercise $(p=0.01)$, but no effect of glutamine supplementation or prior glycogenreducing exercise alone. Bonferroni's post hoc analysis showed a significant difference between GS-D and PS-D $\left({ }^{*}, p<0.05\right)$. Error bars indicate SEM.

Fig. 4. Interaction effects of glutamine supplementation and prior glycogen-reducing exercise on absolute change in a) relative $C D 8^{+}$cell count, b) relative $C D 4^{+}$ cell count, and c) $C D 4^{+}: C D 8^{+}$ratio post-exercise.

WBC counts and the severity of stress, ${ }^{17,20}$ with the exception that our results do not illustrate an association between neutrophil or total lymphocyte count and glycogen status. Also, our results are in agreement with previous studies, that neither total WBC, nor differential WBC counts post-exercise were significantly influenced by glutamine supplementation. ${ }^{36}$ However, if glutamine supplementation is not considered as a main effect (groups GS-R and GS-D) but only in the context of the more severe condition of simulated stage racing with insufficient glycogen supplementation, a different conclusion is reached.

Our results indicate an interaction effect of glutamine and prior glycogen-reducing exercise on T-cell subpopulation distribution, so that the magnitude of exercise-induced decrease in the $C D 4^{+}: C D 8^{+}$ratio is significantly greater after glutamine supplementation when exercise is started in a state of reduced glycogen availability. A possible explanation is that glutamine indirectly affected the $\mathrm{CD} 4^{+}: \mathrm{CD}^{+}$ratio by enhancing DHEAS release. DHEAS is known to increase interleukin-2 (IL-2) secretion, ${ }^{7}$ which enhances the $T_{H} 1$ response, ${ }^{31}$ which includes enhanced differentiation and proliferation of $\left(\mathrm{CD}^{+}\right)$cytotoxic and suppressor T-cells to improve cell-mediated immunity. Therefore, while glutamine may not have a direct effect on immune cell function, it may be indirectly beneficial to cell-mediated immune function.

\section{Conclusion}

The results show that supplementation with glutamine before exercise may have an indirect anticortisol effect by increasing the DHEAS response to exercise. However, this possible effect requires validation in a larger subject group. In addition, 


\begin{tabular}{|c|c|c|c|c|c|c|}
\hline & $\begin{array}{l}\text { Reference range for } \\
\text { general population at rest }\end{array}$ & Time point & GS - D & GS - R & PS - D & PS-R \\
\hline \multirow[t]{2}{*}{ White blood cells } & $4000-11000$ cells/ul & Pre & $5470 \pm 530$ & $5500 \pm 600$ & $5320 \pm 530$ & $5250 \pm 640$ \\
\hline & & Post & $11610 \pm 2530^{*}$ & $7600 \pm 970$ & $9320 \pm 2030$ & $6960 \pm 780$ \\
\hline \multirow[t]{2}{*}{ Neutrophils } & $2000-7500 \mathrm{cells} / \mathrm{ul}$ & Pre & $2920 \pm 460$ & $2950 \pm 490$ & $2800 \pm 550$ & $2860 \pm 490$ \\
\hline & & Post & $8420 \pm 2410^{+}$ & $5260 \pm 690$ & $6300 \pm 1930$ & $4480 \pm 650$ \\
\hline \multirow[t]{2}{*}{ Monocytes } & $0-800$ cells/ul & Pre & $530 \pm 50$ & $530 \pm 30$ & $470 \pm 20$ & $510 \pm 30$ \\
\hline & & Post & $730 \pm 120$ & $510 \pm 70$ & $630 \pm 120$ & $510 \pm 30$ \\
\hline \multirow[t]{2}{*}{ Lymphocytes } & $1000-4000$ cells/ul & Pre & $1800 \pm 200$ & $1800 \pm 190$ & $1870 \pm 300$ & $1730 \pm 290$ \\
\hline & & Post & $2310 \pm 280$ & $1710 \pm 350$ & $2260 \pm 510$ & $1830 \pm 290$ \\
\hline \multirow[t]{2}{*}{$\mathrm{CD}^{+}$} & $1100-1700$ cells/ul & Pre & $1248 \pm 143$ & $1201 \pm 150$ & $1396 \pm 208$ & $1197 \pm 197$ \\
\hline & & Post & $1590 \pm 220$ & $1179 \pm 216$ & $1498 \pm 292$ & $1238 \pm 229$ \\
\hline \multirow[t]{2}{*}{ Relative $\mathrm{CD}^{+}{ }^{+}$} & $\%$ total lymphocytes & Pre & $69.5 \pm 3.9$ & $66.3 \pm 3.6$ & $75.0 \pm 2.7$ & $69.4 \pm 3.9$ \\
\hline & & Post & $67.4 \pm 5.3$ & $68.5 \pm 4.2$ & $68.2 \pm 4.3$ & $65.6 \pm 4.2$ \\
\hline \multirow[t]{2}{*}{$\mathrm{CD}^{+}$} & $700-1100$ cells/ul & Pre & $748 \pm 99$ & $692 \pm 87$ & $726 \pm 118$ & $760 \pm 142$ \\
\hline & & Post & $768 \pm 109$ & $650 \pm 116$ & $782 \pm 146$ & $748 \pm 137$ \\
\hline \multirow[t]{2}{*}{ Relative $\mathrm{CD} 4^{+}$} & $\%$ total lymphocytes & Pre & $41.2 \pm 1.5$ & $38.9 \pm 3.1$ & $39.1 \pm 1.5$ & $43.7 \pm 2.2$ \\
\hline & & Post & $32.1 \pm 1.0$ & $38.1 \pm 2.4$ & $36.2 \pm 2.4$ & $39.4 \pm 1.2$ \\
\hline \multirow[t]{2}{*}{$\mathrm{CD}^{+}$} & $500-900$ cells/ul & Pre & $415 \pm 66$ & $429 \pm 92$ & $498 \pm 93$ & $393 \pm 59$ \\
\hline & & Post & $573 \pm 52$ & $364 \pm 48$ & $506 \pm 105$ & $418 \pm 69$ \\
\hline \multirow[t]{2}{*}{ Relative $\mathrm{CD}^{+}$} & $\%$ total lymphocytes & Pre & $23.4 \pm 3.0$ & $23.2 \pm 3.5$ & $26.4 \pm 3.3$ & $23.2 \pm 2.5$ \\
\hline & & Post & $25.4 \pm 2.6$ & $22.4 \pm 2.3$ & $23.1 \pm 2.3$ & $22.7 \pm 2.4$ \\
\hline \multirow[t]{2}{*}{$\mathrm{CD}^{+}: \mathrm{CD}^{+}$} & $1.0-1.5$ & Pre & $1.88 \pm 0.21$ & $1.87 \pm 0.30$ & $1.61 \pm 0.21$ & $2.01 \pm 0.24$ \\
\hline & & Post & $1.33 \pm 0.18$ & $1.77 \pm 0.22$ & $1.60 \pm 0.14$ & $1.78 \pm 0.18$ \\
\hline
\end{tabular}

Bonferroni's post hoc analysis: * Value significantly increased from baseline: $p<0.05$; Tendency for increase from baseline: $p=0.06$.

GS-D = glutamine-supplemented, prior glycogen-reducing exercise; GS-R = glutamine-supplemented, glycogen-replete and rested; PS-D = placebo-supplemented, prior glycogen-reducing exercise; PS-R = placebo-supplemented, glycogen-replete and rested.

glutamine was associated with a decreased $\mathrm{CD} 4^{+}: \mathrm{CD} 8^{+}$ratio in response to exercise in a glycogen-depleted state.

\section{Acknowledgements}

The authors would like to thank EAS International for the donation of the L-glutamine used as supplement in this study.

\section{RefERENCES}

1. Araneo BA, Shelby J, Li GZ, Ku W, Daynes RA. Administration of dehydroepiandrosterone to burned mice preserves normal immunologic competence. Arch Surg 1993; 128: 318-25

2. Biolo G, Toigo G, Ciocchi B, et al. Metabolic response to injury and sepsis: changes in protein metabolism. Nutrition 1997; 13: 52-7S.

3. Castell LM, Newsholme EA. The relation between glutamine and the immunodepression observed in exercise. Amino Acids 2001; 20: 49-61.

4. Castell LM, Poortmans JR, Newsholme EA. Does glutamine have a role in reducing infections in athletes? Eur J Appl Physiol Occup Physiol 1996; 73: $488-90$.

5. Cutolo M, Foppiani L, Prete C, et al. Hypothalamic-pituitary-adrenocortical axis function in premenopausal women with rheumatoid arthritis not treated with glucocorticoids. J Rheumatol 1999; 26: 282-8.

6. Daynes RA, Araneo BA, Dowell TA, Huang K, Dudley D. Regulation of murine lymphokine production in vivo. III. The lymphoid tissue microen- vironment exerts regulatory influences over $T$ helper cell function. $J$ Exp Med 1990; 171: 979-96.

7. Daynes RA, Dudley DJ, Araneo BA. Regulation of murine lymphokine production in vivo. II. Dehydroepiandrosterone is a natural enhancer of interleukin 2 synthesis by helper T cells. Eur J Immunol 1990; 20: 793-802.

8. Deuster PA, Zelazowska EB, Singh A, Sternberg EM. Expression of lymphocyte subsets after exercise and dexamethasone in high and low stress responders. Med Sci Sports Exerc 1999; 31: 1799-1806.

9. Gleeson M, Bishop NC. Elite athlete immunology: importance of nutrition. Int J Sports Med 2000; 21: Suppl 1, S44-50

10. Gleeson M, Blannin AK, Walsh NP, Bishop NC, Clark AM. Effect of low- and high-carbohydrate diets on the plasma glutamine and circulating leukocyte responses to exercise. Int J Sport Nutr 1998; 8: 49-59.

11. Gollnick PD, Piehl K, Saltin B. Selective glycogen depletion pattern in human muscle fibres after exercise of varying intensity and at varying pedalling rates. J Physiol 1974; 241: 45-57.

12. Grinspoon S, Corcoran C, Stanley T, Rabe J, Wilkie S. Mechanisms of androgen deficiency in human immunodeficiency virus- infected women with the wasting syndrome. J Clin Endocrinol Metab 2001; 86: 4120-6.

13. Hammarqvist F, Ejesson B, Wernerman J. Stress hormones initiate prolonged changes in the muscle amino acid pattern. Clin Physiol 2001; 21: 44-50.

14. Hiscock N, Pedersen BK. Exercise-induced immunodepression-plasma glutamine is not the link. J Appl Physiol 2002; 93: 813-22.

15. Kunz-Ebrecht SR, Mohamed-Ali V, Feldman PJ, Kirschbaum C, Steptoe A. Cortisol responses to mild psychological stress are inversely associated with proinflammatory cytokines. Brain Behav Immun 2003; 17: 373-83. 
16. Miller AL. Therapeutic considerations of L-glutamine: a review of the literature. Alternative Medicine Review 1999; 4: 239-48.

17. Natale VM, Brenner IK, Moldoveanu AI, Vasiliou P, Shek P, Shephard RJ Effects of three different types of exercise on blood leukocyte count during and following exercise. Sao Paulo Medical Journal 2003; 121: 9-14.

18. Nehlsen-Cannarella SL, Fagoaga OR, Nieman DC, et al. Carbohydrate and the cytokine response to $2.5 \mathrm{~h}$ of running. J Appl Physiol 1997; 82: 1662-7.

19. Nieman DC. Immune response to heavy exertion. J Appl Physiol 1997; 82: $1385-94$

20. Nieman DC, Miller AR, Henson DA, et al. Effects of high- vs moderateintensity exercise on natural killer cell activity. Med Sci Sports Exerc 1993; 25: 1126-34

21. Nieman DC, Nehlsen-Cannarella SL, Markoff PA, et al. The effects of moderate exercise training on natural killer cells and acute upper respiratory tract infections. Int J Sports Med 1990; 11: 467-473.

22. Parry-Billings M, Baigrie RJ, Lamont PM, Morris PJ, Newsholme EA. Effects of major and minor surgery on plasma glutamine and cytokine levels. Arch Surg 1992; 127: 1237-40.

23. Parry-Billings $M$, Budgett $R$, Koutedakis $Y$, et al. Plasma amino acid concentrations in the overtraining syndrome: possible effects on the immune system. Med Sci Sports Exerc 1992; 24: 1353-8.

24. Rohde T, MacLean DA, Klarlund PB. Glutamine, lymphocyte proliferation and cytokine production. Scand J Immunol 1996; 44: 648-50.

25. Rohde T, MacLean DA, Pedersen BK. Effect of glutamine supplementation on changes in the immune system induced by repeated exercise. Med Sci Sports Exerc 1998; 30: 856-62.

26. Rohde T, Ullum H, Rasmussen JP, Kristensen JH, Newsholme E, Pedersen BK. Effects of glutamine on the immune system: influence of muscular exercise and HIV infection. J Appl Physiol 1995; 79: 146-50.

27. Rowbottom DG, Keast D, Goodman C, Morton AR. The haematological, biochemical and immunological profile of athletes suffering from the overtraining syndrome. Eur J Appl Physiol 1995; 70: 502-9.
28. Salehian B, Kejriwal K. Glucocorticoid-induced muscle atrophy: mechanisms and therapeutic strategies. Endocr Pract 1999; 5: 277-81.

29. Shephard RJ, Shek PN. Athletic competition and susceptibility to infection. Clin J Sport Med 1993; 3: 75-7.

30. Shinkai S, Watanabe S, Asai H, Shek PN. Cortisol response to exercise and post-exercise suppression of blood lymphocyte subset counts. Int $J$ Sports Med 1996; 17: 597-603.

31. Smith LL. Overtraining, excessive exercise, and altered immunity: is this a T helper-1 versus T helper-2 lymphocyte response? Sports Med 2003; 33: $347-64$

32. Stapelfeldt B, Schwirtz A, Schumacher YO, Hillebrecht M. Workload demands in mountain bike racing. Int J Sports Med 2004; 25: 294-300.

33. Straub RH, Schuld A, Mullington J, Haack M, Scholmerich J, Pollmacher $\mathrm{T}$. The endotoxin-induced increase of cytokines is followed by an increase of cortisol relative to dehydroepiandrosterone (dhea) in healthy male subjects. J Endocrinol 2002; 175: 467-74.

34. Strauss JDW, Myburgh K, Kruger A, Smith C, Robson P. Pre-exercise glutamine supplementation could prevent decreases in postexercise serum glutamine following a single bout of intensive exercise. South African Journal of Sports Medicine 2001; 8: 12-6.

35. Villette JM, Bourin $P$, Doinel $C$, et al. Circadian variations in plasma levels of hypophyseal, adrenocortical and testicular hormones in men infected with human immunodeficiency virus. J Clin Endocrinol Metab 1990; 70: 572-7.

36. Walsh NP, Blannin AK, Bishop NC, Robson PJ, Gleeson M. Effect of oral glutamine supplementation on human neutrophil lipopolysaccharide-stimulated degranulation following prolonged exercise. Int J Sport Nutr Exerc Metab 2000; 10: 39-50.

37. Walsh NP, Blannin AK, Robson PJ, Gleeson M. Glutamine, exercise and immune function. Links and possible mechanisms. Sports Med 1998; 26: 177-91.

38. Wisniewski TL, Hilton CW, Morse EV, Svec F. The relationship of serum DHEA-S and cortisol levels to measures of immune function in human immunodeficiency virus-related illness. Am J Med Sci 1993; 305: 79-83.

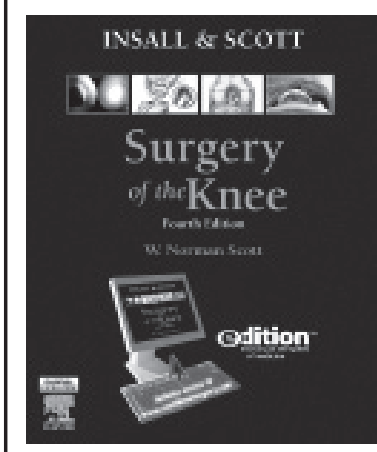

Health \& Medical

Publishing Group

Insall \& Scott's

Surgery of the Knee

e-dition, 4th edition

Text with Continually

Updated Online

Reference, 2-Volume Set

By W. Norman Scott, MD, Clinical Professor of Orthopaedic Surgery, Albert Einstein College of Medicine, Bronx, NY; Director, Insall Scott Kelly Institute for Orthopaedics and Sports Medicine, New York, NY

\section{ISBN 0443069611 - Book/Electronic Media Churchill Livingstone - Published January 2006}

Health \& Medical Publishing Group

Private bag X1, Pinelands, 7430

Tel: 021-6578200 Fax: 021-6834509

e-mail: carmena@hmpg.co.za

brents@hmpg.co.za

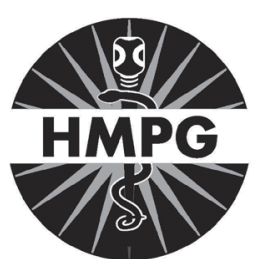

\section{Health \& Medical Books}

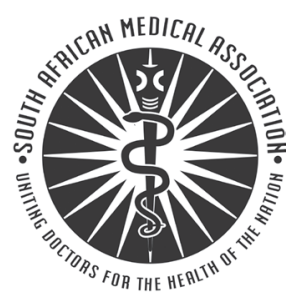

